\title{
HIPPARCOS DATA DISTRIBUTION TO THE COMMUNITY
}

\author{
M.A.C. PERRYMAN, \\ European Space Agency, Astrophysics division \\ ESTEC, Postbus 299 \\ NL - 2200 AG NOORDWIJK aan zee \\ The Netherlands
}

\begin{abstract}
The Hipparcos satellite generates a considerable quantity of 'raw' data: approximately $1 \mathrm{Gbit}$ per day, or some $1000 \mathrm{Gbits}$ over its anticipated lifetime. The Hipparcos mission follows usual practices of having elements of the observing programme selected after an invitation for proposals to the general astronomical community, as well as a strong scientific interest in the data exploitation from amongst the individuals working within the scientific consortia related to the observing programme definition and the data analysis. However, an unusual feature of this mission is the fact that the elemental observations cannot be 'separated out' and distributed to the proposers or the consortia 'principal investigators'. Instead, the data analysis teams will work towards the construction of a single, agreed-upon, catalogue containing the final astrometric and photometric star parameters.

In formulating the rules for the distribution of the reduced Hipparcos data, the following considerations had to be kept in mind: (i) the members of the scientific teams working on the observing programme definition and the data analysis had to retain the opportunity of benefitting scientifically from their involvement; (ii) similar considerations had to apply to the principal investigators of the approved observing programmes, selected in 1982; (iii) while consideration could be given to distributing early data to these parties, appropriate efforts would have to be made to ensure that unvalidated data would not be distributed prematurely - the data validation is expected to be a difficult and delicate task.

The resulting time-schedules for the planned release of the final Hipparcos data, which are by necessity linked to the duration of the mission, will be described. Assuming an end of mission operations at the end of 1992, the final data is expected to be available to the above individuals by the end of 1994, and to the scientific community in general by the end of 1995 . Continued operation of the satellite beyond this date would have a corresponding impact on the schedule for the data release, both internally and externally.
\end{abstract}

